\title{
Drug-Induced Thrombosis: MR Follow-Up After L-Asparaginase Therapy
}

\author{
Teresa Popolizio, Nicola Sforza, and Daniela Grasso
}

- 8-year-old patient with acute lymphoblastic leukemia.

- Early MR follow-up after L-asparaginase treatment.

\section{Early Imaging After Chemotherapy}
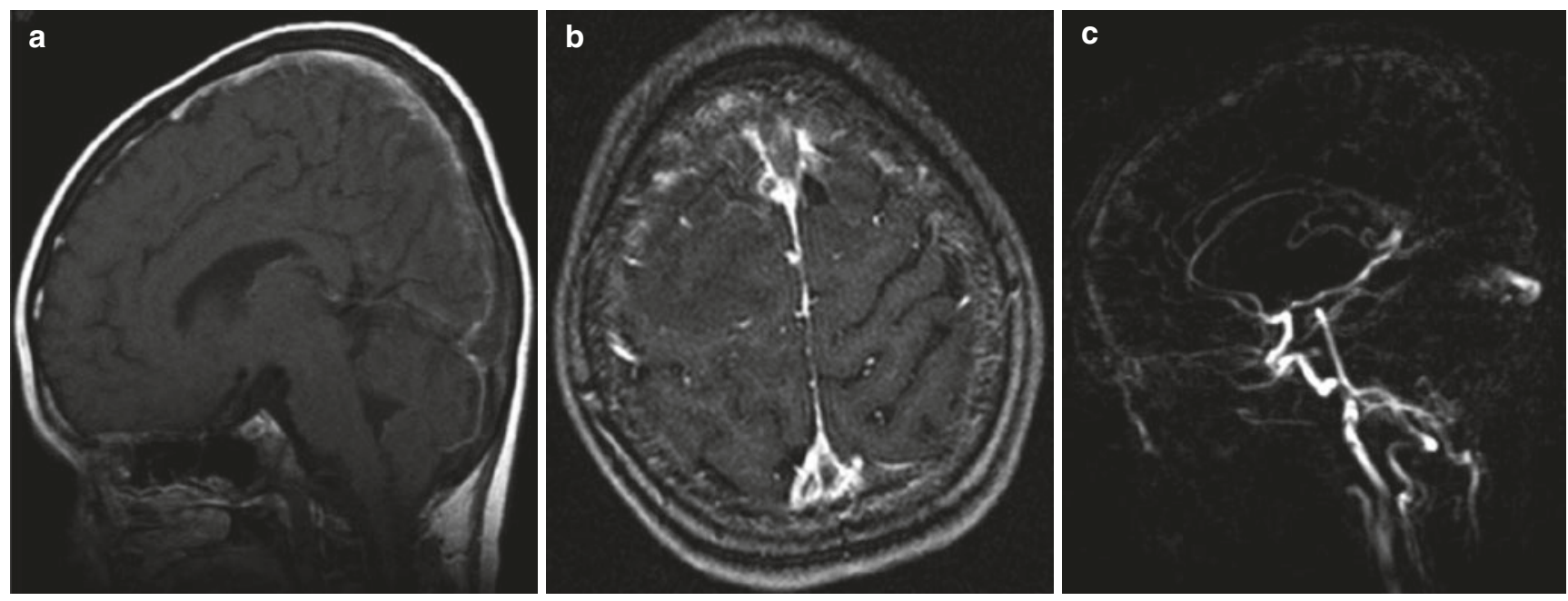

Fig. 52.1 MR SE T1-weighted sequence (a) and MR angiography (3D TOF) in (b), 2D PC MIP in (c). Note the T1 hyperintensity from acute thrombosis with consequent "delta gap" sign in the single partition and no flow signal in the superior sagittal sinus in the MIP image

T. Popolizio $(\bowtie) \cdot$ N. Sforza

Department of Neuroradiology, Fondazione IRCCS "Casa Sollievo della Sofferenza”, San Giovanni Rotondo, FG, Italy

\section{Grasso}

Department of Radiology, Fondazione IRCCS "Casa Sollievo della

Sofferenza”, San Giovanni Rotondo, FG, Italy 